\title{
Tabularia
}

\section{Le cartulaire B du chapitre cathédral de Coutances : histoire d'une résurrection}

The cartulary B of the chapter of Coutances: story of a resurrection

Julie Deslondes-Fontanel

\section{(2) OpenEdition}

Journals

Édition électronique

URL : http://journals.openedition.org/tabularia/499

DOI : 10.4000/tabularia.499

ISSN : 1630-7364

Éditeur :

CRAHAM - Centre Michel de Boüard, Presses universitaires de Caen

Référence électronique

Julie Deslondes-Fontanel, « Le cartulaire B du chapitre cathédral de Coutances : histoire d'une résurrection », Tabularia [En ligne], Les cartulaires normands. Bilan et perspectives de recherche, mis en ligne le 23 décembre 2009, consulté le 19 avril 2019. URL : http://journals.openedition.org/ tabularia/499 ; DOI : 10.4000/tabularia.499 


\section{Le cartulaire B du chapitre cathédral de Coutances: histoire d'une résurrection}

\section{The cartulary $B$ of the chapter of Coutances: story of a resurrection}

Julie DESLONDES-FONTANEL

Archives départementales du Calvados

j.deslondes@cg14.fr

Résumé:

Trois cartulaires furent réalisés par le chapitre de Coutances au XIV siècle, après que «les malheurs du temps» eurent mis en péril la conservation de son chartrier.

Avec la disparition au début du XIX ${ }^{e}$ siècle des cartulaires A et C, le cartulaire B devint un petit monument des archives diocésaines et fit l'objet de plusieurs copies. Ces copies ont permis de réaliser l'édition du manuscrit, détruit lors du bombardement de 1944.

Le cartulaire est d'une ampleur respectable. Il couvre une période chronologique vaste, depuis le duc Guillaume jusqu'au $\mathrm{XV}^{\mathrm{e}}$ siècle, et une aire géographique à la fois étendue et révélatrice des intérêts économiques du chapitre. Sa composition est intéressante, notamment par ce qu'elle révèle de la méthode du compilateur. On regrettera, comme souvent, la stéréotypie de certains actes, mais beaucoup d'autres rencontreront l'intérêt de l'historien.

Mots-clés: chapitre, cathédrale, cartulaire, Coutances, Cotentin, Manche.

\section{Abstract:}

Three cartularies were written by the chapter of Coutances in the XIV th century, as the " misfortunes of time" had endangered the conservation of the charters.

After the disappearance of the cartularies $A$ and $C$ in the early XIX ${ }^{\text {th }}$ century, the cartulary $B$ became a true monument in the eyes of the diocesan archivists. It was therefore copied several times. These copies enabled me to edit the cartulary $B$ after its destruction during the bombings of 1944.

The cartulary is rather voluminous. It covers a wide chronological period, from the duke William to the $X V^{\text {th }}$ century, and a vast geographical area, which also reveals the economic interests of the chapter. Its composition is interesting, especially - by what it says - for the writer's methodology. We may regret that some charters are fairly stereotypical, but many others will be of special interest to historians.

Keywords: chapter, cathedral, cartulary, Coutances, Cotentin, Manche. 


\section{Introduction}

Après la confusion et le relatif désordre religieux du $\mathrm{X}^{\mathrm{e}}$ siècle, l'institution épiscopale est restaurée à Coutances au XI ${ }^{e}$ siècle. L'épiscopat de Geoffroy de Montbray, de 1048 à 1093, voit le retour du chapitre du prieuré Saint-Lô de Rouen à Coutances, et la dédicace de la nouvelle cathédrale en 1056. Le chapitre, constitué à l'époque de sept chanoines, verra progressivement ses prébendes s'accroître, pour arriver à dix-sept chanoines à la fin du XII e siècle et vingt-six en 1250, lors de la visite d'Eudes Rigaud. C'est un chapitre de taille moyenne, comparable à celui de Lisieux en terme de revenus, modeste en comparaison de celui de Bayeux mais nettement plus important que son voisin avranchin ${ }^{1}$.

Son histoire est bien connue pour l'époque moderne grâce aux travaux de Gilles Désiré basés notamment sur les comptes et les délibérations conservés aux Archives diocésaines de Coutances².

À l'inverse, toute étude relative au diocèse de Coutances pour le Moyen Âge semble a priori impossible du fait de la destruction totale des sources originales lors du désastre du 6 juin 1944. En réalité, cet écueil peut être contourné grâce aux nombreuses copies érudites réalisées dès le XVIII siècle, et surtout au XIXe siècle et permettant de connaître un chartrier capitulaire riche, bien documenté, et composé notamment de pas moins de trois cartulaires, tous rédigés à la fin du XIVe siècle. Bien qu'ils ne subsistent pas sous forme originale, l'histoire de ces cartulaires, depuis leur rédaction jusqu'à leur tradition postérieure, et leur composition, est intéressante à plus d'un titre. Le cartulaire B notamment a pu faire l'objet d'une édition scientifique complète grâce aux copies exhaustives conservées ${ }^{3}$. Ces copies sont bien entendu de portée et de qualité inégales, mais elles permettent une étude diplomatique et historique éclairante.

\section{Rédaction, perte et résurrection du cartulaire $B$}

\section{Le contexte de rédaction}

Les archives capitulaires étaient matériellement distinctes des archives de l'évêque, et ce probablement très tôt. On peut supposer que cette séparation s'est faite au moment de l'individualisation des patrimoines de l'évêque et du chapitre, et donc avant $1156^{4}$. L'abbé Hersent l'atteste pour le XIX ${ }^{e}$ siècle 5 . D'ailleurs, les cartulaires du chapitre ne compilent que des actes adressés au chapitre, concernant des conflits dans lesquels il est impliqué ou des biens qu'il acquiert. Les actes adressés à l'évêque sont exceptionnels. Il s'agit le plus souvent d'actes accordés à l'église cathédrale

1. Sur l'histoire et le patrimoine du chapitre au Moyen Âge, cf. Fontanel, Le Cartulaire..., p. 5587.

2. Désiré DIt Gosset, Le Chapitre...

3. Fontanel, Le Cartulaire...

4. Sur cette séparation entre mense capitulaire et mense épiscopale, cf. FonTANEL, 2000.

5. HeRSENT, 1875, p. 203. 
dans son ensemble, avant que le chapitre ne soit véritablement individualisé ${ }^{6}$ ou de confirmations générales des biens de la cathédrale ${ }^{7}$. Au moment de la compilation des cartulaires du chapitre, les actes capitulaires formaient donc un fonds matériellement et intellectuellement distinct de celui de l'évêque.

La réalité du chartrier médiéval du chapitre est très difficile à cerner en-dehors des actes transcrits dans les trois cartulaires du chapitre. Dès le XIX ${ }^{e}$ siècle, en effet, les inventaires des archives diocésaines et départementales attestent de la disparition de la quasi-totalité des actes antérieurs au XIV siècle. Les années 1350-1360, époque à laquelle les trois cartulaires du chapitre furent rédigés, ont été sans doute des années de rupture dans la gestion du chartrier, en raison des troubles liés à la Guerre de Cent ans, à la peste, et au siège de Coutances par les Anglais et les Navarrais en 1356. Dans un aveu de 1540, les chanoines attestent de ces pertes importantes puisqu'ils déclarent à propos de leurs fiefs qu'ils ne peuvent plus retrouver les chartes et les titres qui y sont associés «considéré le temps immemorial, les guerres, hostilités, combustions, ravissements et degradements advenus tant par les Navarrais, Anglois et Bretons qu'autres adversaires es pays et duché de Normandie».

Le chartrier de l'évêché, s'il existait en tant que tel au Moyen Âge, nous est lui entièrement inconnu puisque l'évêché, au contraire du chapitre, ne constitua pas de cartulaires. Il préféra constituer des pouillés, liste des paroisses du diocèse avec leurs patrons et les dîmes perçues sur ces paroisses.

Les trois cartulaires du chapitre ont tous trois été réalisés au cours du $\mathrm{XIV}^{\mathrm{e}}$ siècle. La désignation des cartulaires par des lettres est attestée par Delisle et il précise que celle-ci est utilisée depuis le XVIII siècle. Le terme de Livre blanc du chapitre, quant à lui, est attesté par Mangon du Houguet pour le cartulaire B dès le XVII ${ }^{e}$ siècle et entraîna une longue confusion avec le pouillé épiscopal portant le même nom.

Le cartulaire A renfermait 400 actes depuis 1200 ou environ jusqu'en 1489. Il avait de nombreux actes en commun avec le cartulaire $B^{8}$ qui a pu être pris pour guide. Il est décrit au XVII siècle comme «un ancien registre ou chartrier en parchemin non chiffré couvert blanc ${ }^{9}$. En revanche le cartulaire $C$, décrit au $\mathrm{XVII}{ }^{e}$ siècle comme « un gros registre ou chartrier en parchemin, relié, couvert et fermé à lanières ${ }^{10}$ ", ne comportait aucun acte en commun avec les deux autres cartulaires. Il renfermait 930 actes, c'est-à-dire près du double d'actes que les deux autres recueils, datant du IX ${ }^{\mathrm{e}}$ siècle jusqu'en $1490^{11}$.

Les cartulaires $\mathrm{A}$ et $\mathrm{C}$ ne nous étant connus que par des regestes, il est assez malaisé de comprendre ce qui a poussé le chapitre à réaliser trois manuscrits dont les actes se recoupaient de plus en partie sur une période de temps assez brève.

6. FontANEL, Le Cartulaire..., $\mathrm{n}^{\circ} 289$ et 339.

7. Fontanel, Le Cartulaire..., $\mathrm{n}^{\circ} 135$ et 272.

8. Les actes du cartulaire $\mathrm{A} \mathrm{n}^{\circ}$ 9, 12, 13, 160, 217 à 246, 248, 251, 253, 254, 275, 296, 307 à 341, 343, 345 à 347 sont également dans le cartulaire $\mathrm{B}$.

9. BnF, ms latin 5200 , fol. 75 .

10. BnF, ms latin 5200, fol. 79v.

11. BnF, ms nouvelles acquisitions latines 1018, fol. 4 


\section{Les conditions de la perte et de la résurrection du cartulaire B}

Les cartulaires $A$ et $C$ étaient encore dans les archives diocésaines en 1781 lorsqu'un regeste en fut réalisé ${ }^{12}$. Ils furent probablement détruits à la Révolution, bien qu'aucun élément ne permette d'étayer cette hypothèse, sinon le silence total sur ces manuscrits après cette date.

Il faut en effet être prudent sur les circonstances exactes de leur disparition, en raison du grand désordre des archives au début du XIX ${ }^{e}$ siècle, désordre qui semble avoir été la règle générale dans les dépôts normands à cette époque.

Delisle écrivait que les dépôts lui étaient ouverts « dans des conditions ultralibérales et qui auraient dû [l']effrayer si [il] avait eu plus d'expérience ${ }^{13}$ ». Les archives capitulaires de Coutances subirent de plus de nombreux déménagements dans la première moitié du XIX ${ }^{e}$ siècle. Vers 1840, elles passèrent de la cathédrale aux greniers des communs de l'évêché ${ }^{14}$ et firent alors l'objet d'un premier classement grâce au vicaire général de 1838 à 1861, Mgr. Delamare. C'est à cette époque que l'on retrouve parfois des manuscrits qui avaient été égarés durant les décennies précédentes. On retrouve ainsi à cette période deux exemplaires des pouillés épiscopaux du XIII et XIV e siècle: le Livre noir de l'évêché, retrouvé en $1836^{15}$; le Livre blanc du chapitre, disparu à la Révolution et retrouvé par Delamare dans un sac de parchemins destinés à être utilisés pour confectionner des reliures ${ }^{16}$.

Le cartulaire B, égaré comme d'autres dans le désordre des archives diocésaines, fut également retrouvé par Mgr. Delamare en 1837, dans des circonstances décrites par Gerville ${ }^{17}$ :

Dans un voyage que je fis à Coutances le 9 mai 1837, M. Delamare me fit voir quelques titres des archives épiscopales qui lui avaient paru mériter une attention particulière, quoiqu'il ne pût pas les déchiffrer. Celui qui paraissait le plus important était un gros registre en parchemin [...]. Les personnes employées depuis peu à mettre en ordre les archives de l'évêché avaient cru y voir le Livre blanc, ou registre dressé dans le XIV ${ }^{\mathrm{e}}$ siècle par les ordres de Louis d'Erquery, évêque depuis 1345 jusqu'en 1371, mais c'était une erreur. Le Livre blanc est encore un desideratum et le registre prêté par M. Delamare n'est qu'un recueil ou copie de recueil d'actes, de titres et de notes relatives aux revenus de la cathedrale de Coutances, antérieures à la révolution de 1789. Quoiqu'il en soit, ce recueil considérable ne serait pas sans importance s'il était en bon état, mais malheureusement il a tant souffert de l'injure des temps que la plupart des pages sont mutilées, déchirées et même anéanties.

\footnotetext{
12. Archives diocésaines de Coutances, ms 2.

13. HuARD, 1947, p. 9.

14. Daireaux, 1997, p. 93.

15. HERSENT, 1875, p. 202.

16. Hersent, 1875, p. 205. Sur les manuscrits des pouillés épiscopaux et leur histoire, cf. DÉSIRÉ DIT Gosset, 1997 et Fontanel, Le Cartulaire..., p. 37-41.

17. Musée des Beaux-Arts de Caen, ms 300 du fonds Mancel, fol. 5.
} 
Le cartulaire du chapitre est donc alors perçu comme une déception, un document de moindre valeur que les pouillés de l'évêché, que Delamare et ses collègues cherchent alors activement. Charles Duhérissier de Gerville, ancien émigré, précurseur de la science historique en Normandie et mentor de Léopold Delisle, décide cependant de le copier, pour la première fois au XIX siècle. Il fait cette remarque pertinente: "Peut-être même, vu l'extrême négligence qui règne encore dans les dépôts publics, les choses que je copie aujourd'hui pourront-elles remplacer jusqu'à un certain point celles qui disparaîtraient d'ici à quelques années». Quelques années plus tard, le cartulaire est également cité par Natalis de Wailly dans son rapport de 1839 , dans lequel il souligne encore son mauvais état ${ }^{18}$.

À partir de là, le cartulaire $\mathrm{B}$ devient un petit monument des Archives diocésaines, dont tout le monde perçoit l'intérêt. Il est classé en tête des archives et Nicolas Dubosc le décrit comme le premier manuscrit dans son inventaire des archives diocésaines réalisé en $1841^{19}$.

Il est plusieurs fois copié, soit à des fins scientifiques de "prises de note» - ainsi Delisle ${ }^{20}$, probablement dans le cadre de la préparation de son cartulaire normand publié de 1848 à 1852 - soit à des fins de simple reproduction, dans l'esprit de nos microfilmages et numérisations actuels. Deux ecclésiastiques de Coutances s'y attelleront: Pierre-Auguste Le Cardonnel, premier archiviste diocésain en titre, vers $1860^{21}$; Ernest Fleury au début du $\mathrm{XX}^{\mathrm{e}}$ siècle ${ }^{22}$. Vicaire de chœur et secrétaire de l'évêché de 1890 à 1927, Fleury réalise la seule copie exhaustive du registre ${ }^{23}$. À cette époque, les pouillés ont sans doute déjà été édités par Longnon, et il perçoit certainement l'intérêt de cette source encore inédite. Il est d'ailleurs un copiste infatigable, proche de la graphomanie... Il a réalisé de nombreuses copies intégrales de manuscrits conservés à Coutances, sans aucun commentaire particulier, et ce travail, sans doute très long, a pris une valeur tout à fait particulière quarante ans après...

Le cartulaire B fut également succinctement étudié dans les années 1850 par Léchaudé d'Anisy ${ }^{24}$ qui écrit: «Ce cartulaire a été retrouvé par moi dans les papiers que l'administration départementale avait laissés dans les greniers avant qu'elle fut transférée à Saint-Lô ${ }^{25}$ ». Il fut finalement transféré aux Archives départementales de la Manche, sous la cote G 180, à la suite de la loi de 1905, et c'est là qu'il finit sa carrière en juin 1944.

18. Poulle, 1999, p. 11.

19. Archives diocésaines de Coutances, ms M 3.

20. Bnf, mss nouvelles acquisitions latines 1018-1019.

21. Archives diocésaines de Coutances, $\mathrm{ms} \mathrm{M} 27$ et $\mathrm{M} 28$

22. Archives diocésaines de Coutances, $\mathrm{ms} \mathrm{M} 40$.

23. Cf. Annexe ${ }^{\circ} 1$.

24. Membre de la Société des Antiquaires de Normandie, Léchaudé d'Anisy a beaucoup travaillé dans les dépôts de la Manche. Il a étudié le fonds de Savigny et a été nommé en 1839 par le ministre de l'Instruction publique pour classer les archives de Mortain.

25. BnF, ms latin 10068, fol. 84 . 
Fort heureusement, les copies réalisées au XIX ${ }^{e}$ siècle étaient conservées dans des dépôts épargnés par les bombardements, qu'il s'agisse des archives diocésaines de Coutances (où se trouvaient encore pendant la guerre la majeure partie des archives capitulaires), du musée des Beaux-Arts de Caen pour la copie de Gerville ${ }^{26}$, ou de la Bibliothèque nationale pour celle de Delisle ${ }^{27}$.

\section{Les limites de l'édition d'un manuscrit disparu...}

L'édition du cartulaire B avait d'abord nécessairement pour but, et contrairement à ce qui est la pratique générale aujourd'hui, de reconstituer une partie du chartrier disparu. L'analyse du cartulaire pour lui-même était forcément limitée. Bien des points ne pouvaient être documentés du fait de la destruction du cartulaire lui-même, ainsi que du chartrier. Sur l'ensemble des actes copiés, seul un original était connu, et encore de manière indirecte: il s'agissait, dans le cadre d'un arbitrage entre le chapitre et Troarn, de l'exemplaire envoyé à l'abbaye et conservé dans son fonds aux Archives du Calvados ${ }^{28}$. Toutes les informations qui pourraient découler de la comparaison des originaux avec le cartulaire, pour établir la méthode suivie, le choix des actes par le cartulariste, sa fiabilité dans la copie, manquent donc en partie.

Les copistes dont nous dépendons pour connaître le manuscrit original n'étaient pas de grands diplomates ou paléographes et beaucoup d'éléments leur ont sans doute échappés. Seule exception notable, Léopold Delisle est justement celui dont la copie est la plus succincte, dans la mesure où elle s'apparente surtout à des notes de travail ne retenant que les éléments essentiels du texte.

Cependant, la composition même du cartulaire, quelques éléments apportés par les copistes, la comparaison avec les regestes conservés pour les deux autres cartulaires «frères » et une légère dose d'imagination permettent de faire quelques conjectures...

Nous disposons de peu d'éléments pour ébaucher une analyse codicologique du manuscrit, car les érudits qui se sont intéressés au cartulaire B se sont malheureusement peu souciés de le décrire précisément avant de le recopier. Gerville est le copiste qui nous en donne la description la plus complète, citant la couverture en parchemin jaunâtre, le nombre de feuilles (228), le changement d'écriture à la fin du volume, et la cote indiquée (B ou II) ${ }^{29}$.

Tous les copistes insistent sur le mauvais état du manuscrit et sur l'existence de deux écritures différentes. Dubosc fait une description très complète du foliotage du cartulaire dans son inventaire des archives diocésaines en 1841, et insiste sur la nécessité d'en faire une copie compte-tenu de son mauvais état de conservation $^{30}$. Delisle souligne que les titres du chapitre devaient être déjà en mauvais état

26. Musée des Beaux-Arts de Caen, ms 300 du fonds Mancel.

27. Sur ces copistes et leur travail, cf. Julie FontANEL-DESLONDES, 2007.

28. Arch. dép. 14, H 7780.

29. Musée des Beaux-Arts de Caen, ms 300 du fonds Mancel, fol. 5.

30. Archives diocésaines de Coutances, ms M 3. Cité par Gilles DésIRÉ DIT Gosset, 1997, p. 15. 
au XIV ${ }^{\mathrm{e}}$ siècle et que le rédacteur du cartulaire a dû laisser en blanc les lignes qu'il ne pouvait pas lire ${ }^{31}$.

Léchaudé d'Anisy nous apprend également que «les premières lettres de tous les actes manquent dans le manuscrit parce que le scribe devait sans doute les écrire en encre de couleur ${ }^{32} »$. Le rédacteur avait laissé des lettrines en réserve qui, pour la plupart, n’ont jamais été réalisées. Cette description est confirmée par la copie de Fleury qui laisse très souvent la première lettre des actes en blanc. Quelques lettrines ont malgré tout été réalisées, comme l'atteste Gerville qui reproduit fidèlement des motifs zoomorphes ou anthropomorphes, parfois assez travaillés.

\section{La composition du cartulaire}

\section{La méthode de compilation des actes}

Sur les 360 actes, cinq ont été rédigés deux fois. Il y a donc 355 actes différents. Les actes ont été copiés selon une logique essentiellement géographique et répondant à une certaine progression, du moins jusqu'à l'acte 236 : le cartulaire traite successivement des paroisses du doyenné de Saire, puis de Valognes, d'Orglandes et du Plain, et enfin de Baupte. Dans la suite du cartulaire, il devient plus difficile de dégager une unité: on trouve des actes concernant Le-Mesnil-au-Val ${ }^{33}$, Flottemanville-Bocage ${ }^{34}$, puis un grand nombre d'actes de portée générale. Enfin, la fin du cartulaire concerne en majorité le doyenné de la Chrétienté.

Certains doyennés du diocèse sont plus souvent représentés. Les paroisses $\mathrm{du}$ Cotentin font l'objet de 202 actes, alors que celles du Val-de-Vire ne sont concernées que par 11 actes. Les doyennés les plus documentés sont le doyenné d'Orglandes avec 82 actes puis celui de Valognes, avec 45 actes ${ }^{35}$. La très faible représentation de l'archidiaconé du Val-de-Vire, alors qu'il s'agit d'une province riche et très proche de la ville cathédrale, a de quoi surprendre. Le nombre d'acquisitions faites par le chapitre au nord du diocèse, et singulièrement dans l'archidiaconé du Cotentin, est en revanche impressionnant: 52 actes traitent des rentes ou des terres de la seule paroisse d'Hauteville-Bocage ${ }^{36}$. Cette importance quantitative du nord du diocèse s'explique sans doute par une politique d'acquisition du chapitre, en partie pour profiter des nombreux défrichements qui ont lieu dans cette région au XIII ${ }^{\mathrm{e}}$ siècle. Elle est sans doute moins révélatrice des possessions réelles du chapitre que de sa volonté de s'implanter sur des territoires nouveaux et prometteurs, et de l'impact de cet enjeu sur sa production documentaire. Le cartulaire B du chapitre apparaît ici comme un révélateur de

\footnotetext{
31. BnF, ms nouvelles acquisitions latines. 1018, fol. 3-3v.

32. BnF, ms latin 10068, fol. 84 .

33. Arr. Cherbourg, cant. Tourlaville.

34. Arr. Cherbourg, cant. Tourlaville.

35. FontAnel, Le Cartulaire..., p. 45.

36. Arr. Cherbourg, cant. Saint-Sauveur-le-Vicomte. Fontanel, Le Cartulaire..., actes n 99 à 200.
} 
la construction de l'espace diocésain, au-delà de la zone d'influence immédiate du siège épiscopal, et du rôle du chapitre dans cette construction ${ }^{37}$.

Si le compilateur a donc esquissé un regroupement géographique assez habituel, celui-ci n'est pas systématisé et s'articule avec une organisation par dossier thématique. Le cartulaire rassemble par exemple à la suite d'une charte de donation les actes annexes qui affermissent le chapitre dans ses droits: transactions antérieures, ratification de l'acquisition par l'autorité ecclésiastique ou par les parties concernées, nominations des procureurs ou des arbitres en cas de procès, ratification par la femme du vendeur lorsque le bien provenait de sa dot, etc. De la même manière, les actes concernant les dîmes des novales sont presque tous rassemblés au même endroit ${ }^{38}$.

\section{Répartition des actes par période, par auteur et par langue ${ }^{39}$}

La quasi-totalité des actes du cartulaire B sont antérieurs à 1347. Il y a ensuite un fossé dans la chronologie entre les statuts de 1377 et les onze actes suivants qui terminent le cartulaire et qui sont datés de 1497 à 1514. Il semble donc certain que le cartulaire proprement dit ait été rédigé dans les années 1370-1380, et que les onze derniers actes du cartulaire aient été rédigés dans un deuxième temps, un siècle plus tard.

Sur les 355 actes différents, trois appartiennent avec certitude au XI siècle: ils datent de l'épiscopat de Geoffroy de Montbray qui initie le renouveau du chapitre après son long exil à Rouen ${ }^{40}$. Le développement du chapitre et de sa production documentaire semble ensuite assez lent jusqu'à l'essor de la seconde moitié du XIII ${ }^{e}$ siècle. Vingt-six actes seulement datent du XII ${ }^{e}$ siècle et 234 actes, du XIII siècle dont 39 seulement antérieurs à 1250. Plus de la moitié de la documentation date donc de la seconde moitié du XIII e siècle, ce qui n'a rien pour nous étonner. L'activité du chapitre se ralentit au XIV siècle, peut-être sous l'influence des troubles qui agitent la région: 81 actes sont datés du XIV ${ }^{\mathrm{e}}$ siècle, dont un seulement est postérieur à 1350 .

Les actes s'étendent sur une très longue période et passent progressivement du latin au français. $24 \%$ des actes sont rédigés en français. Le premier date du 30 janvier 1278 (v. st. ${ }^{41}$ mais la langue vernaculaire ne s'impose véritablement qu’à partir du XIVe siècle. Entre 1279 et 1299, seuls dix-neuf actes sur 84 sont écrits en français. Au XIV siècle la situation s'inverse et moins du tiers des actes est rédigé en latin. Aux XV et $\mathrm{XVI}^{\mathrm{e}}$ siècle, un seul acte est rédigé en latin ${ }^{42}$. Classiquement, la langue vernaculaire s'impose d'abord chez les laïcs. Elle est marquée, mais par

37. Sur ces enjeux, cf. MAZEL, 2008.

38. FontANel, Le Cartulaire..., actes $n^{\circ} 241,243,245,246,247,248,249,250,251,253,259,261,262$, $264,265,267,268,269$.

39. Cf. FontAnel, Le Cartulaire..., p. 46-52.

40. Sur cette période, SPEAR, The personnel...

41. Fontanel, Le Cartulaire..., acte $n^{\circ} 27$.

42. Fontanel, Le Cartulaire..., acte $\mathrm{n}^{\circ} 356$. 
intermittence, par des usages locaux: «chatel » pour désigner les bien mobiliers; «cabot» ou «bingue» pour désigner des mesures de céréales; «deile», pour une pièce de terre.

Les laïcs sont les auteurs les plus fréquents, comme dans le cartulaire de Savigny et nombre d'autres cartulaires ecclésiastiques ${ }^{43}$. Parmi eux, il est difficile de distinguer la catégorie des seigneurs. Seuls huit actes mentionnent des domini appartenant à l'aristocratie. La grande majorité des auteurs laïcs ne sont gratifiés d'aucun titre. Ils ont recours à un sigillant s'ils n'ont pas leur propre sceau ou s'ils doutent de sa validité. De plus, ils font très souvent confirmer leurs actes par des actes émis par une institution ecclésiastique. Les femmes apparaissent souvent dans les actes aux côtés de leur mari pour renoncer à leurs droits sur leur dot, mais aussi parfois comme auteurs: dix actes sont rédigés par des veuves disposant de leur propre sceau.

À partir des années 1280-1290, les laïcs font presque toujours appel à une juridiction gracieuse pour l'établissement de leurs actes. Cette fonction n'est assurée que trois fois par les évêques de Coutances ${ }^{44}$. Les officiaux de Coutances et Valognes se substituent rapidement à eux dans cet office. Puis, au début du $\mathrm{XIV}^{\mathrm{e}}$ siècle, les officiers royaux deviennent les principaux acteurs de la juridiction gracieuse: le bailli du Cotentin rédige parfois lui-même les actes mais délègue le plus souvent cet office à son lieutenant, et très vite aux vicomtes. Entre 1300 et 1514, les deux tiers des actes sont passés dans les sièges de vicomtés, principalement celles de Carentan et Coutances. Leur élaboration est généralement déléguée au garde-scel, appelé à Coutances "garde des obligations de la viconté», et le plus souvent assisté par un tabellion, appelé «clerc, attourné».

\section{L'intérêt historique et diplomatique des actes}

Les actes antérieurs à la fin du XII siècle sont particulièrement intéressants. Le cartulaire reproduit en particulier une notice du duc Guillaume ${ }^{45}$, rédigée lors $\mathrm{du}$ rétablissement du chapitre à Coutances après le long exil rouennais. Cet acte, le plus ancien connu pour cette cathédrale, avait sans doute essentiellement un usage interne permettant de résumer les biens et privilèges du chapitre. Il est exceptionnel en Normandie où très peu d'actes de confirmation générale sont conservés pour les chapitres cathédraux au XI ${ }^{\mathrm{e}}$ siècle. Parmi les privilèges pontificaux transcrits, celui de 1146 est également très intéressant ${ }^{46}$. Rédigé à une période importante pour le diocèse, sous l'épiscopat d'Algare, il s'appuie de toute évidence sur la liste établie au $\mathrm{XI}^{\text {e }}$ siècle, mais en opérant cette fois clairement la distinction entre mense capitulaire et mense épiscopale.

\footnotetext{
43. Poulle, 1989.

44. FontAnel, Le Cartulaire..., actes $n^{\circ} 65,136,146$.

45. FontAnel, Le Cartulaire..., acte $\mathrm{n}^{\circ} 340$.

46. Fontanel, Le Cartulaire..., acte $\mathrm{n}^{\circ} 348$. Sur la comparaison entre l'acte de Guillaume de 1056 et le privilège pontifical de 1146, cf. FonTANEL, 2000.
} 
D'un point de vue diplomatique, les actes du XIII siècle présentent un formulaire relativement répétitif. Le cartulaire comprend cependant, par exemple, dix actes passés coram parrochia entre 1258 et $1343^{47}$.

La grande majorité des actes sont de nature économique. Ils enregistrent des transactions, ventes ou dons et reflètent parfaitement les préoccupations essentiellement patrimoniales des chanoines. Au XII e siècle, la plupart des actes concernent des patronages et des droits ecclésiastiques, et surtout des dons ad puram et perpetuam elemosinam caractéristiques de la Réforme grégorienne.

À partir des années 1264-1265, les ventes de rentes constituent la masse des acquisitions. Si l'on s'en tient aux informations du cartulaire, le chapitre acheta surtout des rentes dans l'archidiaconé du Cotentin et plus particulièrement dans le doyenné d'Orglandes et à Hauteville-Bocage. Les acquisitions au nord des Veys sont beaucoup plus rares. Les fondations d'obits sont également nombreuses, qu'il s'agisse des fondations importantes des évêques Hugues de Morville ${ }^{48}$ et Jean $\mathrm{d}^{\prime} E s s e{ }^{49}$, mais aussi d'obits de particuliers à partir des années 1340. La question de la propriété des dîmes des novales de la forêt de Brix donne également lieu à un grand nombre d'actes.

Comme dans la plupart des cartulaires ecclésiastiques, les actes de portée liturgique ou concernant l'organisation du chapitre sont rares. Cependant, deux statuts épiscopaux du XIV ${ }^{e}$ siècle ont été transcrits ${ }^{50}$, ainsi que le règlement du collège des enfants de chœur en $1500^{51}$. On peut également suivre des achats de prébende ou des fondations de chapelles. Enfin, le cartulaire documente les relations parfois conflictuelles entre le chapitre et Jean d'Essey. Il reproduit ainsi trois accords et arbitrages intervenus entre 1256 et 1269 et établissant les obligations et les privilèges de chacun à la suite du conflit de juridiction qui les avait opposés ${ }^{52}$.

\section{Conclusion}

Le travail des érudits passionnés du XIX ${ }^{e}$ siècle, malgré ses lacunes, et l'absence d'informations codicologiques notamment, a empêché la disparition complète d'une source particulièrement importante pour la Basse-Normandie. Il nous permet de spéculer sur les conditions d'élaboration de cartulaires aujourd'hui détruits, et nous donne à voir l'activité d'un chapitre modeste, sans doute peu original mais actif et en perpétuelle mutation.

Le cartulaire en lui-même est révélateur des intérêts du chapitre, de son tropisme pour le nord du diocèse et de son attachement à la construction d'un patrimoine qui s'étend progressivement sur l'ensemble du territoire diocésain.

\footnotetext{
47. Fontanel, Le Cartulaire..., actes $n^{\circ} 83,87,48,53,56,164,204,216,80,169$.

48. FontANEL, Le Cartulaire..., acte $\mathrm{n}^{\circ} 327$.

49. FontANEL, Le Cartulaire..., acte $\mathrm{n}^{\circ} 227$.

50. Fontanel, Le Cartulaire..., actes $\mathrm{n}^{\circ} 242$ et 349 .

51. FontAnel, Le Cartulaire..., acte $\mathrm{n}^{\circ} 358$.

52. Fontanel, Le Cartulaire..., actes $\mathrm{n}^{\circ} 342,343,344$.
} 
D'autres analyses et une meilleure compréhension du contexte de production seraient possibles en comparant plus précisément le cartulaire $B$ aux autres cartulaires connus ou conservés dans le département: cartulaires $A$ et $C$ du chapitre, ou cartulaire de l'abbaye du Vœu par exemple. La conclusion de cet article pourrait donc être que des travaux sont encore possibles sur le Cotentin médiéval malgré la perte d'une grande partie de la documentation originale...

\section{Bibliographie}

DaIREAux, Christiane, «Les divers périls auxquels ont échappé, au cours des siècles, les archives du chapitre de la cathédrale de Coutances», in Chapitres et cathédrales en Normandie, Actes du congrès des sociétés historiques et archéologiques de Normandie (Bayeux, 1996), Annales de Normandie, Caen, 1997, p. 91-96.

DésIRÉ DIT Gosset, Gilles, Le Chapitre cathédral de Coutances aux XVe et XVI siècles, thèse pour le dipl. d'archiviste-paléographe, 1995 (dactyl.); résumé dans École nationale des chartes, positions des thèses..., 1995, p. 75-82.

DÉSIRÉ DIT Gosset, Gilles, «Les Livres noirs et les Livres blancs de l'ancien diocèse de Coutances», Revue de la Manche, t. 39, 1997, p. 7-21.

DÉSIRÉ DIT GossET, Gilles, «Les chanoines du chapitre cathédral de Coutances», Revue de la Manche, t. 41, 1999, p. 17-40.

Fontanel, Julie, Le Cartulaire du Chapitre cathédral de Coutances, Saint-Lô, Archives départementales de la Manche, 2003, 607 p.

FonTANEL, Julie, «La réorganisation religieuse sous Guillaume le Conquérant: le cas de l'église de Coutances», Revue de l'Avranchin, t. 77, 2000, p. 189-208.

Fontanel-DesLondes, Julie «Léopold Delisle et l'érudition dans le Cotentin: l'exemple du chartrier de Coutances", in Léopold Delisle, actes du colloque de Cerisy-la-Salle, Saint-Lô, 2007, p. 205-212.

Hersent, Louis-François, «Le Livre noir du chapitre, le Livre noir de l'évêché et le Livre blanc du chapitre de l'église cathédrale de Coutances», Mémoires de la société académique du Cotentin, t. 1, 1875, p. 198-206.

Huard, Georges, «Léopold Delisle et la Normandie», Cahiers Léopold Delisle, t. 1, 1947, p. 5-29.

MAZEL, Florian, «L'Espace du diocèse dans les cartulaires cathédraux (XIe-XIV ${ }^{e}$ siècle)», in L'Espace du diocèse: genèse d'un territoire dans l'Occident médiéval, Florian MAZEL (dir.), Rennes, Presses Universitaires de Rennes, 2008, p. 367-402.

Poulle, Béatrice, Le chartrier de l'abbaye de Savigny au diocèse d'Avranches, thèse pour le dipl. d'archiviste paléographe, 1989 (dactyl.); résumé dans École nationale des chartes, positions des thèses, 1989, p. 167-171.

Poulle, Emmanuel, «Le transfert du chartrier de Savigny», Revue de l'Avranchin, t. 76, 1999, p. 1-19.

SPEAR, David S., The personnel of the norman cathedrals during the Ducal period, 9111204, coll. Fasti ecclesiae Anglicanae, Londres, Institute of historical research, 2006, XL-344 p. 


\section{Annexes}

Annexe 1: la tradition du cartulaire B

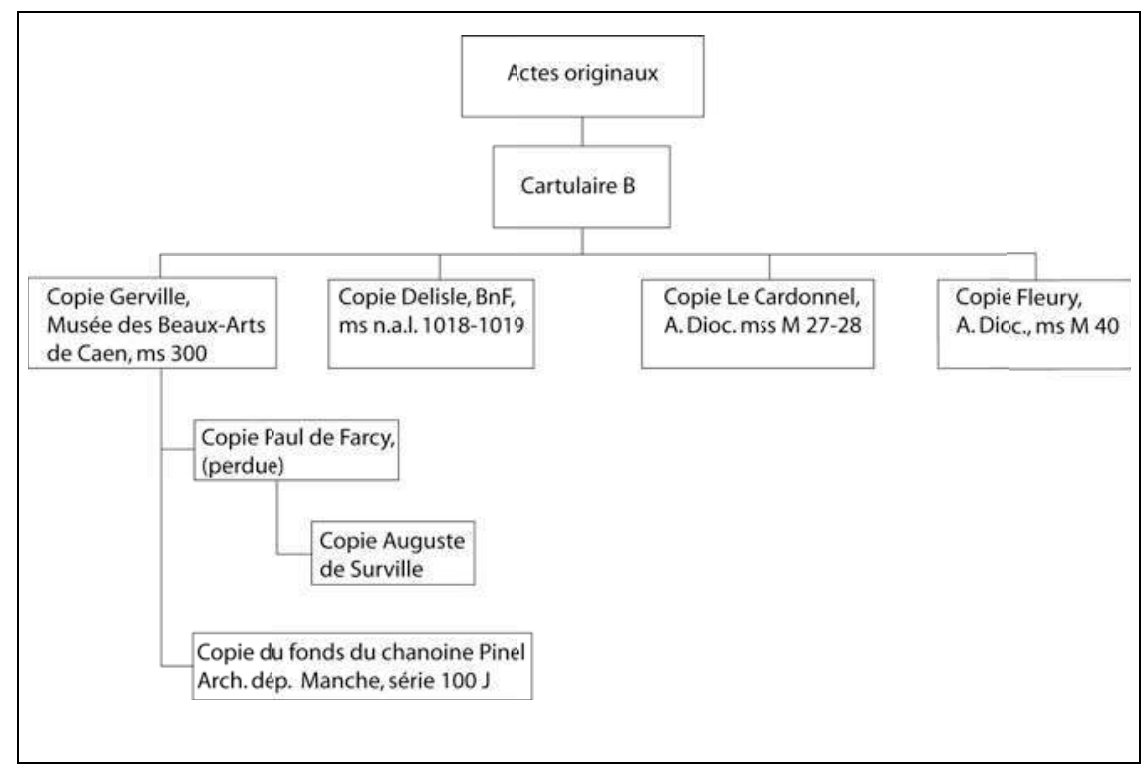

http://www.unicaen.fr/mrsh/craham/revue/tabularia/print.php?dossier=dossier9\&file=04deslondes.xml 
Annexe 2: le cartulaire B du chapitre cathédral de Coutances
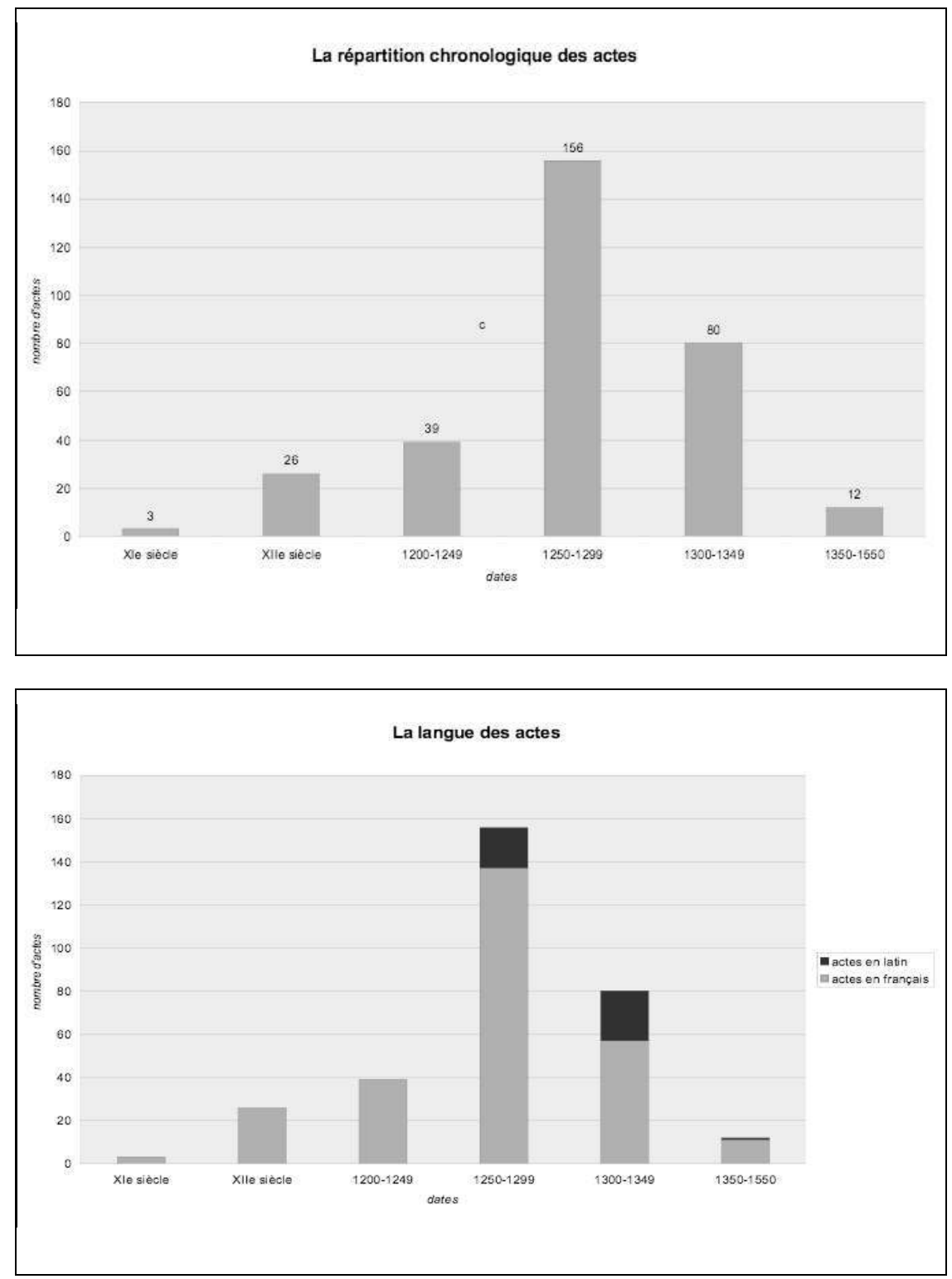

Tabularia «Études», n 9, 2009, p. 71-84, 23 décembre 2009 